\title{
PAPER \\ Compensation of nonlinear fibre impairments in coherent systems employing spectrally efficient modulation formats
}

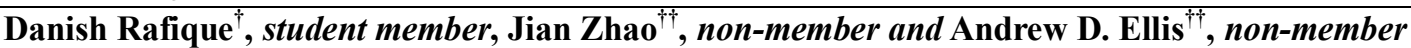

\begin{abstract}
SUMMARY We investigate electronic mitigation of linear and nonlinear fibre impairments and compare various digital signal processing techniques, including electronic dispersion compensation (EDC), single-channel back-propagation (SC-BP) and backpropagation with multiple channel processing (MC-BP) in a ninechannel $112 \mathrm{~Gb} / \mathrm{s}$ PM-mQAM $(\mathrm{m}=4,16) \mathrm{WDM}$ system, for reaches up to $6,320 \mathrm{~km}$. We show that, for a sufficiently high local dispersion, SC$\mathrm{BP}$ is sufficient to provide a significant performance enhancement when compared to EDC, and is adequate to achieve BER below FEC threshold. For these conditions we report that a sampling rate of two samples per symbol is sufficient for practical SC-BP, without significant penalties.

key words: Optical fibre communication, Nonlinear optics, Digital signal processing, coherent communication.
\end{abstract}

\section{Introduction}

With the ever increasing demand for high information rates and current advances in more and more bandwidth intense user-end applications (online gaming, ubiquitous video, etc.), the available transmission capacity for single mode optical fibre is exhausting at a very fast rate and is predicted to reach its theoretical limit [1-2] within a decade $[3,4]$. The information rate in an optical transmission system is limited by the interplay between amplified spontaneous emission (ASE) noise, chromatic dispersion (CD), and Kerr fibre nonlinearity. The recent revival of coherent detection with the availability of high speed digital signal processing (DSP) technologies has enabled electronic mitigation of these effects $[5,6]$, as an alternative to traditional techniques like dispersion management [7-9] and optical phase conjugation (OPC) [10-12]. In particular, electronic signal processing using back-propagation with inverse fibre parameters or time inversion has been applied to the compensation of intraand inter-channel nonlinearities [13-18]. Whilst OPC requires the location of an optical processing device close to the mid-point of the system, back-propagation may be located at the transmitter [13] or receiver [14-18], and is thus significantly more compatible with the demand of a dynamic optical network. This practicality is recently accelerated by extensive studies to simplify

Manuscript received October 31, 2010.

Manuscript revised xxxx xx, 2010.

The authors are with Photonic Systems Group, Tyndall National Institute and Department of Electrical Engineering $^{\dagger} /$ Physics $^{\dagger \dagger}$, University College Cork, Dyke Parade, Prospect Row, Cork, Republic of Ireland. danish.rafique@tyndall.ie, +353-21-490-4866 the back-propagation calculation in the electrical domain [19-23]. At the same time, coherent detection has enabled the use of spectrally efficient advanced modulation formats, e.g. polarization multiplexed quadrature amplitude modulation (PM-mQAM) [24-26]. Numerous studies have been carried out recently to investigate the robustness of PM-mQAM against both linear and nonlinear propagation effects [27-29].

In this paper we extend our previous report [23], investigating the maximum potential increase in transmission performance for long haul and ultra longhaul $112 \mathrm{~Gb} / \mathrm{s}$ PM-mQAM transmission systems employing electronic dispersion compensation (EDC), single-channel back-propagation (SC-BP), and backpropagation with multiple channels (MC-BP). We show that by optimizing the dispersion map, back-propagation based on only single-channel computation is sufficient to achieve $6,320 \mathrm{~km}(1,600 \mathrm{~km})$ at bit error rate (BER) of $10^{-3}$ for PM-4QAM (PM-16QAM). Moreover, we address the back-propagation complexity associated with the analogue-to-digital converter sampling rate and show that a sampling rate of 2 samples per symbol shows negligible performance penalty (compared to e.g. 16 samples per symbol) for SC-BP.

\section{Back-propagation model}

Back-propagation processes the received signals by launching them into a virtual fibre with link parameters of opposite-sign values of those in the transmission channel. In practice, this method can be implemented by calculating the nonlinear Schrödinger equation (NLSE) governing the signal propagation through the fibre channel at the receiver. The NLSE governing the forward-propagation (transmission channel) is written as [30]:

$$
-\frac{\partial E}{\partial z}-\frac{\alpha}{2} E-\frac{i \beta_{2}}{2} \frac{\partial^{2} E}{\partial t^{2}}+\frac{\beta_{3}}{6} \frac{\partial^{3} E}{\partial t^{3}}+i \gamma|E|^{2} E=0
$$

where $E$ is the envelope of the optical field, $\beta_{j}$ represents the $j^{\text {th }}$-order dispersion, $\alpha$ is the fibre loss coefficient, $\gamma$ is the nonlinear parameter, and $t$ is the retarded time. For back-propagation, this equation is numerically solved using the split step Fourier method (SSFM) [31], where $\alpha, \beta$ and $\gamma$ are set to be the exact opposite values to those in the transmission fibre, and the optical amplifiers (power gain $G$ ) are replaced with optical attenuators 
(power gain $1 / G$ ). In the absence of noise, it can be proved that back-propagation method can fully compensate the linear and nonlinear fibre impairments to arbitrary precision, provided that sufficient steps are taken in each fibre span. Note that this method is similar to that of propagating the signal along an identical link after optical phase conjugation [10-12], but with added benefits that the impact of third order (and other odd ordered) dispersion may be accommodated. It is also noted that although precise calculation of the SSFM for back-propagation is still complicated, especially for multi-channel cases and higher sampling rate, various methods have been proposed to simplify the electronic signal processing [19-23]. On the other hand, it is also desirable to analyze the technique in a system level to simplify the required specifications when this method is used in optical transmission systems and is optimized together with other parameters in the system, e.g. dispersion map.

\section{Simulation setup}

The transmission link comprised nine WDM channels in two scenarios: either $112 \mathrm{~Gb} / \mathrm{s}$ PM-4QAM or PM16QAM modulation schemes were investigated at a baud-rate of $28 \mathrm{Gbaud}$ or $14 \mathrm{Gbaud}$, respectively. The channel spacing was set to be $50 \mathrm{GHz}$ (or $25 \mathrm{GHz}$ ) with the centre $112 \mathrm{~Gb} / \mathrm{s}$ PM-mQAM channel operating at $1550 \mathrm{~nm}$. The optical transmitters consisted of continuous wave $(\mathrm{CW})$ lasers, followed by two nested Mach-Zehnder modulator (MZM) structures for $\mathrm{x}$ - and y-polarization states. For all the carriers, both the polarization states were modulated independently using de-correlated (100 bits) $2^{13}$ pseudo-random bit sequences (PRBSs). Each PRBS was de-multiplexed separately into multi-level output symbol streams which were used to modulate an in-phase carrier and a quadrature phase carrier, respectively. The simulation conditions ensured 16 samples per symbol, with $2^{13}$ total simulated symbols (see Fig. 1a). Note that third order dispersion was not considered in this study.

\begin{tabular}{llll} 
& \multicolumn{4}{c}{ Table 1 } & Fibre parameters. \\
\cline { 2 - 5 } & Loss & Dispersion & Nonlinearity \\
\cline { 2 - 4 } SMF & $0.2(\mathrm{~dB} / \mathrm{km})$ & $20^{\dagger}(\mathrm{ps} / \mathrm{nm} . \mathrm{km})$ & $1.4(1 / \mathrm{W} \cdot \mathrm{km})$ \\
DCF & $0.5(\mathrm{~dB} / \mathrm{km})$ & $-80(\mathrm{ps} / \mathrm{nm} \cdot \mathrm{km})$ & $2.8(1 / \mathrm{W} \cdot \mathrm{km})$ \\
\cline { 2 - 3 } & †unless mentioned otherwise & &
\end{tabular}

The nine $112 \mathrm{~Gb} / \mathrm{s}$ PM-mQAM signals were multiplexed and propagated over a number of uniform dispersion maps using dual-stage erbium doped fibre amplifiers (EDFAs). The link comprised $N \mathrm{x} 80 \mathrm{~km}$ spans of standard single mode fibre (SSMF) for transmission. The dispersion compensating fibre (DCF) was located at the amplifier mid-stage (see Table I for fibre parameters), as shown in Fig. 1b. Each amplifier stage was modelled with a $4.5 \mathrm{~dB}$ noise figure and the total power was kept constant for each stage [32] resulting in a slight variation in the signal launch power of successive amplifiers. Where appropriate, the input power into DCF was kept 5 $\mathrm{dB}$ lower than that of the SSMF. Unless otherwise specified, an optical attenuator was included at the midstage access point to keep the total mid-stage loss including the DCF and attenuator to be a fixed value of $10 \mathrm{~dB}$. This is typically required to ensure gain flatness, but in this paper it subsequently helped to isolate the influence of nonlinearities for direct comparison under different dispersion maps and confirmed that the benefits of an uncompensated dispersion map (when backpropagation was employed) were associated with minimization of nonlinear effects, rather than just an improvement in optical signal-to-noise ratio (OSNR). In addition, we included the case without this attenuator for the dispersion map without any DCF. Polarization mode dispersion and laser line-width were neglected in our simulations in order to determine limitations arising purely from fibre nonlinearities, dispersion and noise. All the numerical simulations were carried out using VPItransmissionMaker ${ }^{\circledR}$ v.8.3, and MATLAB ${ }^{\circledR}$ v.7.9.

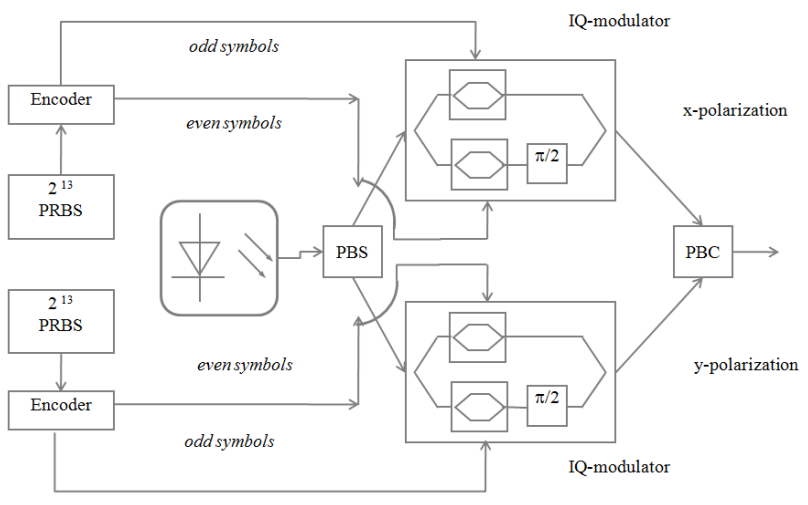

(a)

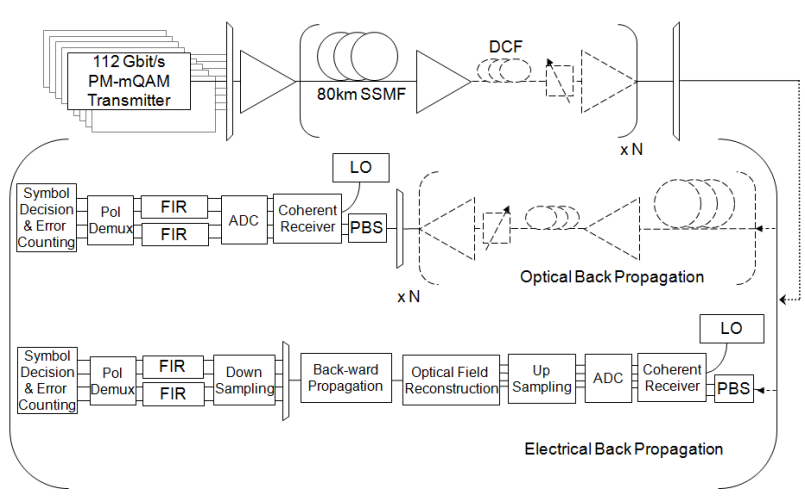

(b)

Fig. 1 Simulation setup a) PM-mQAM $(m=4,16)$ transmitter, b) WDM PM-mQAM transmission system

After fibre transmission, the received fields were stored, and subsequently processed with a number of different receiver configurations. Back-propagation was 
numerically implemented for a variety of bandwidths, representing both SC-BP, and back-propagation with two or more neighbouring channels (MC-BP). To achieve this, in addition to single-channel baseband electronic domain back-propagation (Fig. 1b), we also emulated back-propagation using a simulated optical link in order to establish fundamental performance limits. The optical back-propagation was implemented as follows: an ideal optical filter with bandwidth varying from $25 \mathrm{GHz}$ to $500 \mathrm{GHz}$ was used to select between one to nine channels for back-propagation. Back-propagation of the selected channels was implemented by placing a fibre link with the configuration identical to the forward transmission link but opposite parameter values, where the sample rate and precision were also the same as those in the transmission links. This enabled the required optimum back-propagation bandwidth, and the impact of dispersion map to be established. After back-propagation the channel of interest $\left(5^{\text {th }}\right.$ WDM channel) was demultiplexed, mixed with a local oscillator (LO) laser and
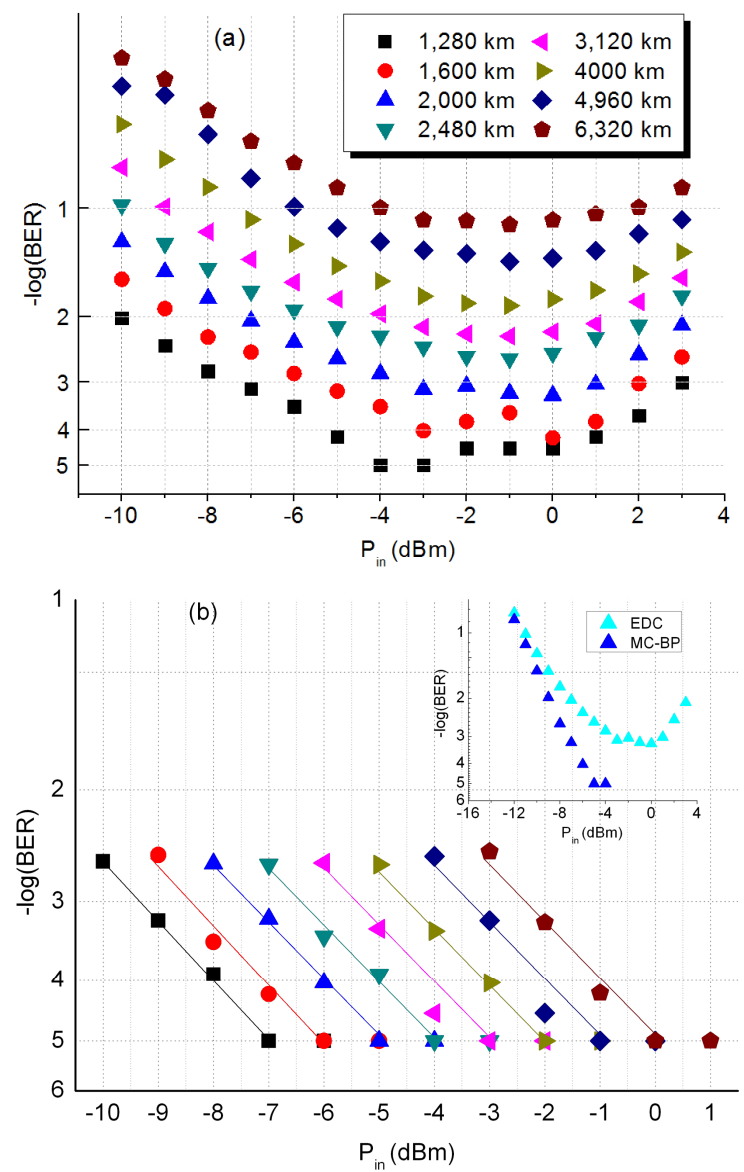

Fig. 2 BER of a nine-channel $112 \mathrm{~Gb} / \mathrm{s}$ PM-4QAM transmission system without inline optical dispersion compensation as a function of launch power per channel per span. We plot various transmission reaches for (a) EDC alone for residual dispersion compensation and (b) MC-BP. Also shown in the inset is a direct comparison of the performance of the two compensation strategies after $2,000 \mathrm{~km}$. down-converted to give a complex baseband electrical signal using a balanced receiver. The baseband signal was then low pass filtered and synchronously sampled at 2 samples per symbol followed by digital field reconstruction from the in-phase and quadrature samples. In order to emulate the practical approach of baseband electronic domain back-propagation, we performed single-channel digital back-propagation (where ninechannel PM-4QAM transmission was employed), where back-propagation was performed after coherent fielddetection by reconstructing the optical field, followed by SSFM based solution of coupled NLSE (dualpolarization) in electrical domain. Note that DBP was implemented with same step-size and precision as forward propagation, and in both forward and backpropagation calculations, the upper bound on the stepsize was set to be $1 \mathrm{~km}$ and the step length was chosen adaptively during the integration along the fiber based on the condition that in each step the nonlinear effects must change the phase of the optical field by no more than 0.05 degrees. In all cases the residual chromatic dispersion for various dispersion maps was compensated with a receiver amplitude response implemented using finite impulse response (FIR) filters [5] (fractionallyspaced taps) which were adapted using a constant modulus algorithm. Polarization de-multiplexing was then performed using a modified butterfly structure. In this structure, samples from the two polarizations were interleaved, allowing for the use of two independent 5 tap filters instead of the usual four filters, in contrary to the traditional butterfly structure [34]. Note that no carrier phase recovery was performed since laser linewidth was neglected in this study. The symbol decisions were made, allowing the performance to be assessed by direct error counting.

\section{Results and Discussions}

Fig. 2 shows the performance for a system without inline dispersion compensation (and no mid-stage loss) as a function of signal launch power per channel per span by using (a) only EDC and (b) MC-BP $(500 \mathrm{GHz})$ for 112 $\mathrm{Gb} / \mathrm{s}$ PM-4QAM. In the figures, the transmission distance is varied from $1,080 \mathrm{~km}$ up to $6,320 \mathrm{~km}(16$ spans to 79 spans). Fig. 2(a) shows that the evolution of BER with signal launch power using EDC alone is clearly consistent with the well-known optimum launch power phenomenon for optical transmission, where at lower launch powers, the system performance is limited by noise and the performance peaks at a certain optimum launch power. After that, the BER rolls off due to increased accumulation of fibre nonlinearities. It can be seen that below 2,000 km, BER below forward error correction (FEC) threshold $\left(10^{-3}\right)$ can be achieved. For longer distances, the continued linear accumulation of amplified spontaneous emission with distance would require increased launch power to achieve an adequate 
OSNR. However increased accumulation of self phase and cross phase modulation also scale linearly with distance, constraining the optimum launch power to an approximately constant value, and consequently achievable BER performance decreases with distance. Note that BER of $1 \mathrm{e}-5$ indicates that no errors were detected, implying that if one error was detected, the 3sigma confidence interval should be $[0,1.91 \mathrm{e}-4]$ and $[0$, 6e-5] for 4QAM and 16QAM, respectively, and BER of 1e-5 should be extrapolated as such.

In contrast, with ideal reversal of nonlinear fibre impairments using MC-BP, BER lower than or equal to $10^{-5}$ can be achieved, given that a longer PRBS is used, not only for shorter distances but also for distances as long as $6,320 \mathrm{~km}$, verifying the compensation capability of back-propagation for both linear and nonlinear transmission impairments, i.e. a substantial performance improvement is achieved in comparison to dispersion compensation only. The inset of Fig. $2 b$ directly compares the performance with and without fullbandwidth back-propagation as a function of launch power for a transmission distance of 2,000 km. For launch powers below $-10 \mathrm{dBm}$ (OSNR limited), identical BER are observed for both with and without compensation of nonlinearities. However, between -5 and $-10 \mathrm{dBm}$, whilst a direct analysis of Fig. 2a would suggest that the system is still in noise-limited operating region, a substantial improvement in performance may be obtained using back-propagation. The impact of fibre nonlinearities in this regime may be anticipated by calculating the mean accumulated nonlinear phase shift (nine channels), which is between 0.7 and 2.2 radians in this region. It is worth mentioning here that a finite laser linewidth consideration (neglected in this study) would affect the optimum launch powers observed, however this can be avoided by employing recent carrier phase recovery algorithms, e.g. for the worst-case scenario employing 16QAM, 1.4 MHz linewidth can be tolerated [33].

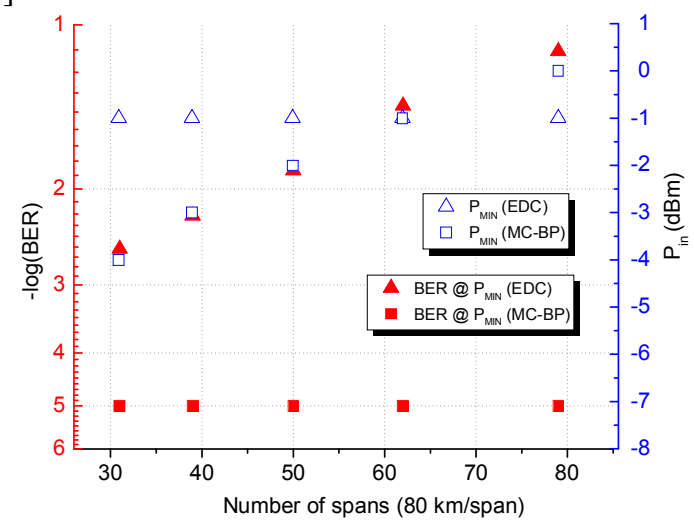

Fig. 3 Minimum achievable BER (left axis, red) and required $P_{\min }$ per channel per span, observed for the given system (right axis, blue) versus number of fibre spans by using EDC alone (triangles) and fullbandwidth back-propagation (squares) for a system without inline optical dispersion compensation (PM-4QAM).
The trends observed in Fig. 2 are summarized in Fig. 3, which plots the minimum required launch power to achieve lowest potential BER for the given system $\left(\mathrm{P}_{\min }\right)$ and the respective BER for EDC alone and MC-BP. In the case that only dispersion is compensated, the BER performance monotonically decreases with the increasing distance, and the $\mathrm{P}_{\min }$ required to achieve the maximum performance remains constant for any distance. It is important to consider here that the constant $\mathrm{P}_{\min }$ corresponds to nonlinear transmission dominated by cross phase modulation (XPM) effects, which increases linearly with distance. This is in contrast to systems with smaller channel spacing, such as orthogonal frequency division multiplexed systems, where four-wave mixing (FWM) is the dominant nonlinear impairment, giving a quadratic nonlinear penalty. In such a case, $\mathrm{P}_{\min }$ would vary as the square root of the length [35]. Conversely, by using full-bandwidth back-propagation, required $\mathrm{P}_{\min }$ to achieve the lowest BER for the given system, increases linearly with the fibre length whilst the BER is constant. In particular, for systems below 5,000 km in length, a lower launch power is required for the back-propagation system than that required for dispersion compensation, with a launch power reduction of around $5 \mathrm{~dB}$ at 1,600 $\mathrm{km}$. The overall performance enhancement enabled by the back-propagation method including the fibre nonlinearity compensation clearly grows monotonically with transmission distance.
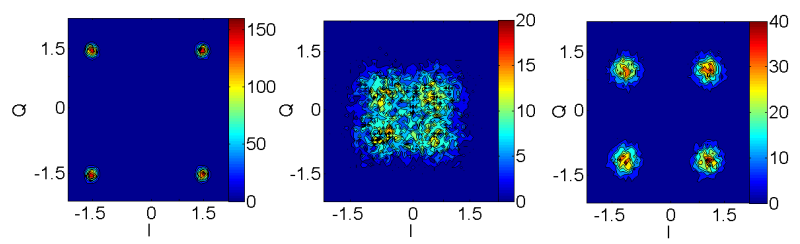

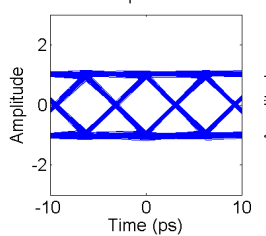

(a)

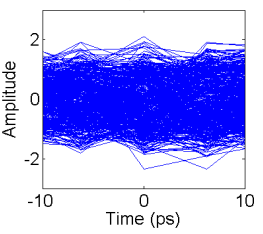

(b)

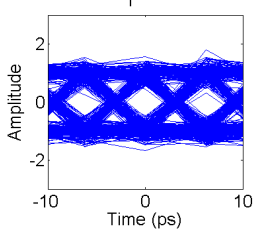

(c)
Fig. 4 Constellation maps (top) and demodulated eye diagrams (bottom) of $112 \mathrm{~Gb} / \mathrm{s}$ PM-4QAM signals for a) the back-to-back signal; b) using only EDC after $6,320 \mathrm{~km}$; c) using full-bandwidth backpropagation after $6,320 \mathrm{~km}$

The benefits of back-propagation for increasing the transmission performance in systems limited by fibre nonlinearities are further illustrated in Fig. 4, which shows the constellation maps (top) and demodulated eye diagrams (bottom) of the $5^{\text {th }}$ WDM channel for both back-to-back (Fig. 4a) and after 6,320 km (Fig. 4b,c). It is clearly visible that for electronic dispersion compensation alone (Fig. 4b), the eye is completely closed, and the constellation diagram is severely degraded. On the other hand, the quality of both the eye diagram and the constellation plots are significantly 

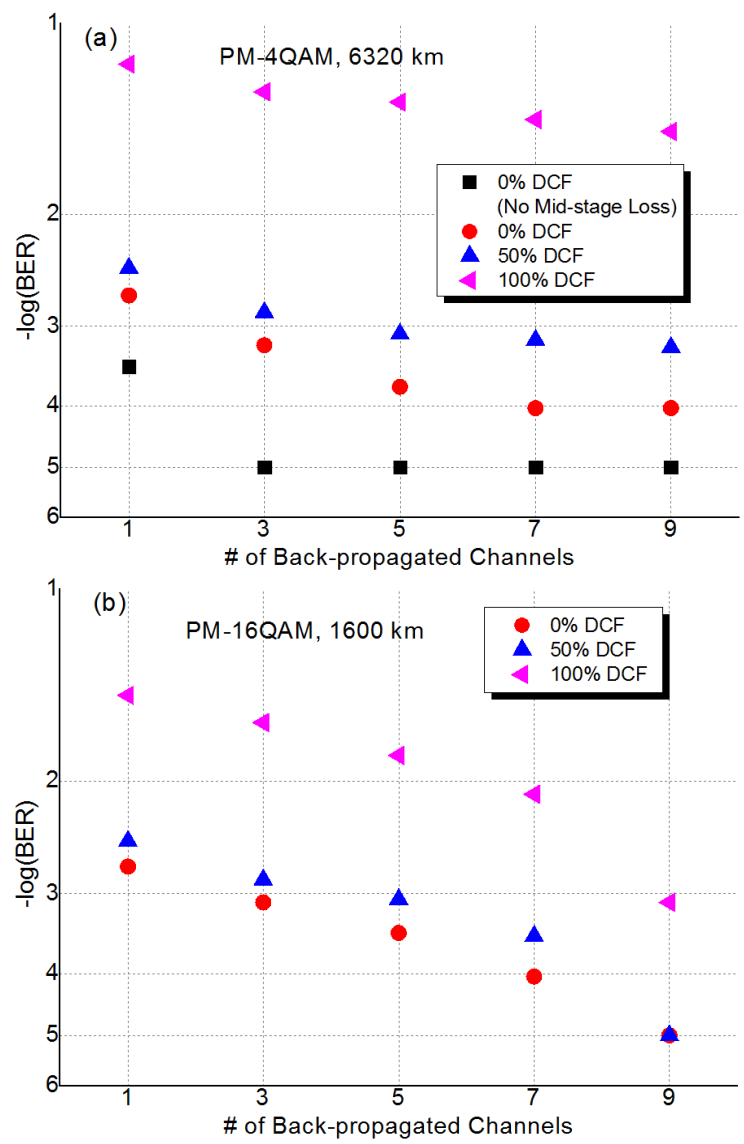

Fig. 5 Performance of back-propagation versus the number of channels included in the back-propagation computation for different dispersion maps in a nine-channel $112 \mathrm{~Gb} / \mathrm{s}$ a) PM-4QAM, b) PM-16QAM system after $6,320 \mathrm{~km}$ and $1,600 \mathrm{~km}$ transmission, respectively.

enhanced by MC-BP due to the compensation of nonlinear fibre impairments. The degradation with respect to the back-to-back case represents the impact of amplified spontaneous emission at the minimum required launch power.

The above investigation is based on a system without inline optical dispersion compensation, clearly proving the performance improvement provided by MC-BP. However, MC-BP is not practical in electronic-domain implementations, which is limited by the bandwidth constraints of the electronic interfaces and sampling rate of the analogue-to-digital converter. Clearly, a reduction of the number of WDM channels which are used for the back-propagation computation would simplify the implementation, but would sacrifice the compensation capability for inter-channel nonlinearities. On the other hand, proper dispersion management can minimize the effects of inter-channel nonlinearities, and consequently has a significant impact on the trade-off between performance (favouring a large compensation bandwidth) and complexity (favouring a low compensation bandwidth). We address this issue by studying the performance of back-propagation as a function of the number of channels included in back- propagation for different dispersion maps. This is illustrated in Fig. 5, which shows that the achievable BER for a $6,320 \mathrm{~km}(1,600 \mathrm{~km})$ nine-channel $112 \mathrm{~Gb} / \mathrm{s}$ PM-4QAM (PM-16QAM) system as a function of the number of adjacent channels considered in the backpropagation computation, for a range of dispersion maps. The figure shows that, at the distances studied, for both PM-4QAM and PM-16QAM, the dispersion maps with highest residual dispersion per span (RDPS) have a weak dependency on the number of back-propagated channels. Indeed, BER around FEC threshold can be achieved even with SC-BP for maps which compensate for less than $50 \%$ of the inline dispersion. This observation can be attributed to weak quasi-phase matching due to the high residual dispersion; thereby intra-channel nonlinearities dominate the transmission which could be reversed by SC-BP. Fig. 5a also shows that for the dispersion map with no inline dispersion compensation for PM-4QAM, the performance of MC-BP is strongly dependent on the number of channels in the back-propagation calculation, although beyond $350 \mathrm{GHz}$ (seven channel backpropagation), the system OSNR results in a BER floor of $10^{-4}$. If the OSNR is improved by removing the midstage loss, the performance can be improved to a BER of $10^{-5}$ even by considering only two additional channels in the back propagation calculation. Fig. 5 also shows that in an OSNR optimised system without DCF, the benefits from both OSNR improvement and minimized interchannel effects enable a BER less than $10^{-3}$ even using single-channel back-propagation.

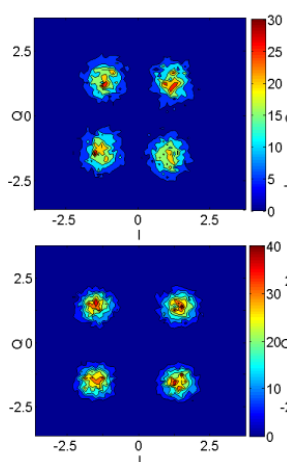

(a)

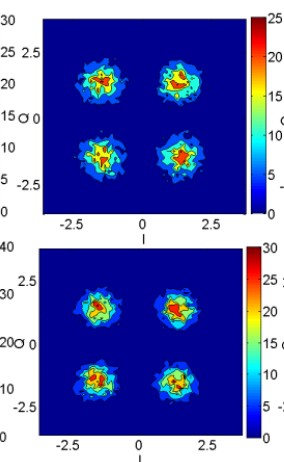

(b)
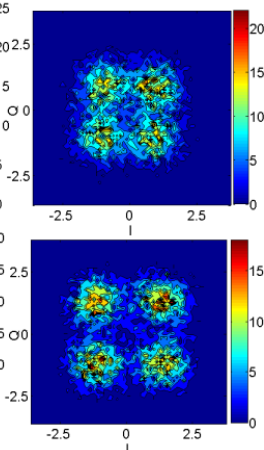

(c)

Fig. 6 Constellation diagrams after single-channel (top) and fullbandwidth (bottom) back-propagation of a nine-channel 112 $\mathrm{Gb} / \mathrm{s} /$ channel PM-4QAM transmission system after $6,320 \mathrm{~km}$ a) for dispersion maps with $0 \%$ inline compensation; b) $50 \%$ inline compensation; c) $100 \%$ inline compensation.

On the other hand, for $100 \%$ inline dispersion compensation map (zero residual dispersion per span), we see that for PM-4QAM transmissions the performance may only be slightly improved even after extending the back-propagation calculation to full bandwidth (nine channels), and the BER is always worse than $5 \times 10^{-2}$, regardless of the number of channels included in back-propagation. We attribute this effect to 

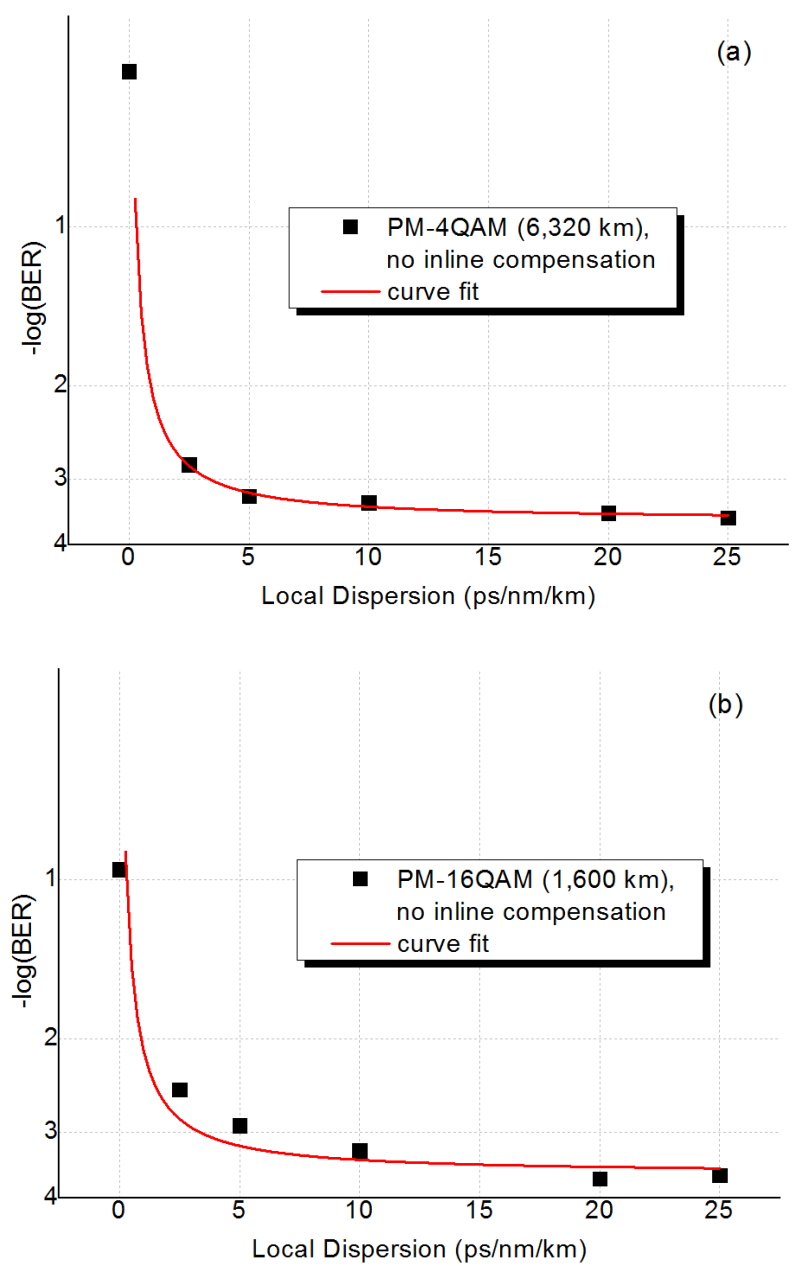

Fig. 7 BER as a function of local dispersion for a nine-channel WDM $112 \mathrm{~Gb} / \mathrm{s}$ a) PM-4QAM, b) PM-16QAM, with no inline dispersion compensation (no mid-stage loss), after single-channel backpropagation. Data (symbols), Line (curve fit)

the irreversible incorporation of ASE into the signal by strongly quasi phase matched signal-ASE nonlinearities, where the origin of such effects could either be XPM [36] or FWM [37]. On the contrary, for the PM-16QAM system using $100 \%$ inline dispersion compensation map, we observe that the overall system performance is strongly dependent on the number of neighbouring channels back-propagated and a BER improvement of $\sim 1.5$ decades is observed for MC-BP in contrast to SCBP. The difference in behaviour for the two transmission schemes considered for $100 \%$ inline dispersion compensation map is due to a better OSNR for the PM16QAM signal at $1,600 \mathrm{~km}$ resulting in smaller random distortions arising from nonlinear signal-ASE interaction. Furthermore, the appearance of a strong dependence on the number of back-propagated channels for PM-16QAM can be attributed to stronger phase matching due to the narrower channel spacing. Consequently, in a PM16QAM system, although this dispersion map can restore BER above FEC threshold when using full-band backpropagation, it is not suitable for practical scenarios.
Constellation plots for full-bandwidth and single-channel back-propagation over $0 \%, 50 \%, 100 \%$ dispersion maps are shown in Fig. 6 (a)-(c) for PM-4QAM. The potential benefits of SC-BP with a sufficiently strong dispersion map are confirmed by Fig. 6a and 6b, where negligible degradation is observed. These results confirm that interchannel effects are reduced by increased RDPS, permitting nonlinearity compensation by backpropagation carrying only a limited portion of the system bandwidth. Furthermore, since SC-BP fully compensates for intra-channel effects, the residual dispersion is no longer constrained by management of peak-to-average power ratio, and arbitrarily high RDPS may be beneficial.

Fig. 7 further illustrates the dependence of local link dispersion considering a system with no inline dispersion compensation (and no mid stage loss), when only SC-BP is implemented. It is clear from the figure that with increasing local dispersion (approx. $>\sim 5 \mathrm{ps} / \mathrm{nm} / \mathrm{km}$ for PM-4QAM, and $>\sim 10 \mathrm{ps} / \mathrm{nm} / \mathrm{km}$ for PM-16QAM at same penalty), the phase matching bandwidth of the system continues to reduce and the rate of degradation due to uncompensated inter-channel nonlinearity is reduced, allowing for SC-BP to restore excellent BER performance.

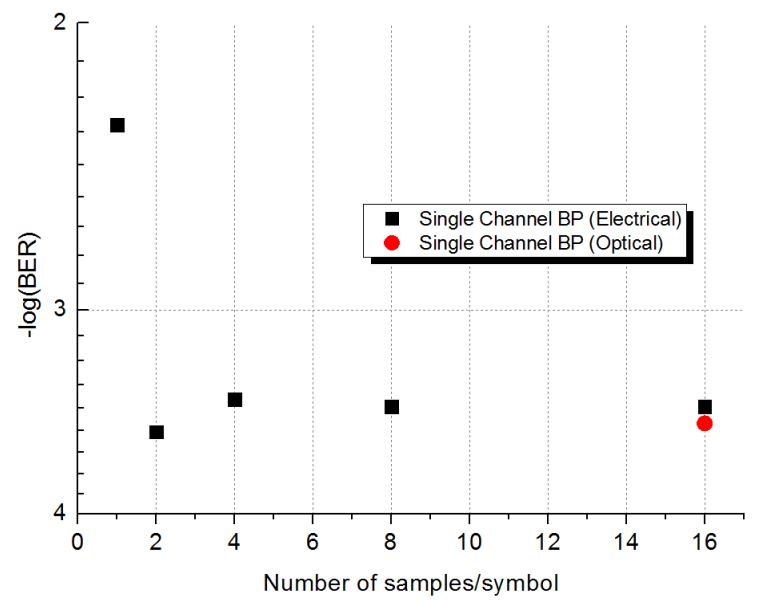

Fig. 8 BER of the electrically implemented single-channel backpropagation method as a function of the sampling rate of the ADC for a nine-channel PM-4QAM system with $0 \%$ inline dispersion compensation (and no mid-stage loss)

Having demonstrated that for a 28 GBaud system with no inline dispersion compensation and a dispersion of $>\sim 5 \mathrm{ps} / \mathrm{nm} / \mathrm{km}$, SC-BP is sufficient to achieve BER below FEC threshold, we may consider the design of the back-propagation electronics. Fig. 8 shows the performance of the electrically implemented singlechannel digital back-propagation method versus the sampling rate of the ADC, where the nine-channel PM4QAM transmission system at $112 \mathrm{~Gb} / \mathrm{s}$ had no inline optical dispersion compensation and no mid-stage loss was considered. It is clearly evident from the figure that 2 samples/symbol (or 56 Gsamples/s) is sufficient to 
avoid performance penalties, which is achievable by current microelectronics technologies, where 56 Gsamples/s ADC with 8 bit resolution is available [38]. Also, negligible performance difference is observed between the optical back-propagation and electrical back-propagation scenarios employing 16 samples/symbol.

\section{Conclusions}

We have demonstrated that the bandwidth constraints of back-propagation method for fibre nonlinearity compensation can be minimized given a sufficiently high RDPS; and the use of single-channel back-propagation is demonstrated to be sufficient to significantly enhance the performance for nine-channel $112 \mathrm{~Gb} / \mathrm{s}$ PM-mQAM $(\mathrm{m}=4,16)$ WDM transmission for long haul and ultra long haul links. In particular, it has been shown that for dispersion map without inline optical dispersion compensation, electrical-domain single-channel backpropagation with 2 samples per symbol can achieve significant improvement compared to electronic dispersion compensation, and is sufficient to achieve BER below FEC threshold.

\section{Acknowledgments}

This work was supported by Science Foundation Ireland under Grant 06/IN/1969, and the authors would like to thank Dr. Marco Forzati and Jonas Mårtensson (Acreo $\mathrm{AB}$, Sweden) for the valuable discussions.

\section{References}

[1] P.P. Mitra, and J. B. Stark, "Nonlinear limits to the information capacity of optical fibre communications," Nature, vol. 411(6841), pp. 1027-1030, 2001.

[2] R.J. Essiambre, "Exploring capacity limits of fibre-optic communication systems," Proc. 34th European Conference on Optical Communications ECOC, no. We.1.E.1, 21-25 Sept 2008.

[3] A.D. Ellis, J.Zhao, D.Cotter, "Approaching the non-Linear Shannon limit," IEEE J. Lightwave Technol., vol.28, no.4, pp.423-433, 2010.

[4] E. Desurvire, "Capacity demand and technology challenges for lightwave systems in the next two decades," IEEE J. Lightwave Technol., vol. 24, pp. 4697-4710, 2006.

[5] S.J. Savory, "Compensation of fibre impairments in digital coherent systems," Proc. 34th European Conference on Optical Communications ECOC, no. Mo.3.D.1, 21-25 Sept. 2008.

[6] E. Ip, A.P.T Lau, D.J.F. Barros, and J.M. Kahn, "Coherent detection in optical fiber systems," Opt. Express, vol.16, pp.753791, 2008.

[7] A.D. Ellis, J.D. Cox, D. Bird, J. Regnault, J.V. Wright, W.A. Stallard, "5 Gbit/s soliton propagation over $350 \mathrm{~km}$ with large periodic dispersion coefficient perturbations using erbium doped fibre amplifier repeaters,” Electronics Letters, vol.27, no.10, pp.878-880, 1991.
[8] I. Morita, K. Tanaka, N. Edagawa, M. Suzuki, "Impact of the dispersion map on long-haul $40 \mathrm{Gbit} / \mathrm{s}$ single-channel soliton transmission with periodic dispersion compensation," in Proc. Optical Fiber Communication Conference OFC, no. 62/FD1-1, 1999.

[9] E. Pincemin, A. Tan, A. Bezard, A. Tonello, S. Wabnitz, J.D. Ania-Castañòn, and S. Turitsyn, "Robustness of $40 \mathrm{~Gb} / \mathrm{s}$ ASK modulation formats in the practical system infrastructure," Opt. Express, no.14, pp.12049-12062, 2006.

[10] W. Pieper, C. Kurtzke, R. Schnabel, D. Breuer, R. Ludwig, K. Petermann, H.G. Weber, "Nonlinearity-insensitive standard-fibre transmission based on optical-phase conjugation in a semiconductor-laser amplifier," Electronics Letters, vol.30, no.9, pp.724-726, 1994.

[11] S. Watanabe, M. Shirasaki, "Exact compensation for both chromatic dispersion and Kerr effect in a transmission fiber using optical phase conjugation,” IEEE J. Lightwave Technol., vol.14, no.3, pp.243-248, 1996.

[12] S.L. Jansen, D.v.d. Borne, B. Spinnler, S. Calabrò, H. Suche, P.M. Krummrich, W. Sohler, G.-D. Khoe, and H. de Waardt, “Optical phase conjugation for ultra long-haul phase-shift-keyed transmission,” IEEE J. Lightwave Technol., vol.24, pp.54, 2006.

[13] C. Weber, C.-A. Bunge, and K. Petermann, "Fiber nonlinearities in systems using electronic predistortion of dispersion at 10 and 40 Gbit/s," IEEE J. Lightwave Technol., vol.27, pp.3654-3661, 2009.

[14] E. Ip, "Nonlinear compensation using backpropagation for polarization-multiplexed transmission," IEEE J. Lightwave Technol., vol.28, pp.939-951, 2010.

[15] E. Yamazaki, F. Inuzuka, K. Yonenaga, A. Takada, M. Koga, "Compensation of interchannel crosstalk induced by optical fiber nonlinearity in carrier phase-locked WDM system," IEEE Photonics Technology Letters, vol.19, no.1, pp.9-11, 2007.

[16] G. Goldfarb, M.G. Taylor, Guifang Li, "Experimental demonstration of fiber impairment compensation using the splitstep infinite impulse response method," IEEE/LEOS Summer Topical Meetings, 2008 Digest of the, no.ME3.1, pp.145-146, 2123 July 2008.

[17] X. Li, X. Chen, G. Goldfarb, Eduardo Mateo, I. Kim, F. Yaman, and $\mathrm{G}$. Li, "Electronic post-compensation of WDM transmission impairments using coherent detection and digital signal processing," Opt. Express, vol.16, pp.880-888, 2008.

[18] S.J. Savory, G. Gavioli, E. Torrengo, P. Poggiolini, "Impact of interchannel nonlinearities on a split-step intrachannel nonlinear equalizer," IEEE Photonics Technology Letters, vol.22, pp.673675,2010

[19] S. Oda, T. Tanimura, T. Hoshida, C. Ohshima, H. Nakashima, Z. Tao, J.C. Rasmussen, "112 Gb/s DP-QPSK transmission using a novel nonlinear compensator in digital coherent receiver," in Proc. Optical Fiber Communication Conference OFC, no. OThR6, 2125 March 2009.

[20] L.B. Du, and A.J. Lowery, "Improved single channel backpropagation for intra-channel fiber nonlinearity compensation in long-haul optical communication systems," Opt. Express, vol.18, pp.17075-17088, 2010.

[21] T. Tanimura, T. Hoshida, S. Oda, T. Tanaka, C. Ohsima, Zhenning Tao, J.C. Rasmussen, "Systematic analysis on multi-segment 
dual-polarisation nonlinear compensation in $112 \mathrm{~Gb} / \mathrm{s}$ DP-QPSK coherent receiver," Proc. 35th European Conference on Optical Communications ECOC, no. 9.4.5, 20-24 Sept. 2009.

[22] C. Jonas Geyer, C.R.S. Fludger, T.Duthel, C.Schulien, and B.Schmauss; "Simple automatic nonlinear compensation with low complexity for implementation in coherent receivers," Proc. 36th European Conference on Optical Communications ECOC, no. P3.02, 19-23 Sept. 2010.

[23] D. Rafique, J. Zhao, A.D. Ellis, "Impact of dispersion map management on the performance of back-propagation for nonlinear wdm transmissions," Proc. OptoElectronics and Communications Conference OECC, no. 00107, 5-9 July 2010.

[24] IEEE P802.3ba $40 \mathrm{~Gb} / \mathrm{s}$ and $100 \mathrm{~Gb} / \mathrm{s}$ Ethernet, "Plenary meeting March 2010, "Available: http://www.ieee802.org/3/ba/public/mar10/index.htm, accessed: $1 / 10 / 10$.

[25] P.J. Winzer, et al., "Spectrally efficient long-haul optical networking using 112-Gb/s polarization-multiplexed 16-QAM," IEEE J. Lightwave Technol., vol.28, pp.547, 2010.

[26] G. Gavioli et al., "100Gb/s WDM NRZ-PM-QPSK long-haul transmission experiment over installed fiber probing non-linear reach with and without DCUs," Proc. 35th European Conference on Optical Communications ECOC, no. 3.4.2, 20-24 Sept. 2009.

[27] S.J. Savory et al., "Electronic compensation of chromatic dispersion using a digital coherent receiver," Opt. Express, vol.15, pp.2120-2126, 2007.

[28] C. Laperle et al., "Wavelength division multiplexing (WDM) and Polarization mode dispersion (PMD) performance of a coherent 40Gbit/s dual-polarization quadrature phase shift keying (DPQPSK) transceiver", in Proc. Optical Fiber Communication Conference OFC, no. PDP16, 2007.

[29] P. Serena, N. Rossi, A. Bononi, "Nonlinear penalty reduction induced by PMD in 112 Gbit/s WDM PDM-QPSK coherent systems," Proc. 35th European Conference on Optical Communications ECOC, no. 10.4.3, 20-24 Sept. 2009.

[30] G. P. Agrawal, Nonlinear fiber optics, Academic Press, 2007.

[31] J. Leibrich; W. Rosenkranz, "Efficient numerical simulation of multichannel WDM transmission systems limited by XPM," IEEE Photonics Technology Letters, vol.15, no.3, pp.395-397, 2003.

[32] A.P.T Lau and J.M. Kahn, "Power profile optimization in phasemodulated systems in presence of nonlinear phase noise," IEEE Photonics Technology Letters, vol.18, no.23, pp.2514-2516, 2006.

[33] T. Pfau, S. Hoffmann, R. Noe, "Hardware-Efficient Coherent Digital Receiver Concept With Feedforward Carrier Recovery for $M$-QAM Constellations," IEEE J. Lightwave Technol., vol.27, no.8, pp.989-999, 2009.

[34] C.R.S. Fludger, et al. "Coherent equalization and POLMUX-RZDQPSK for robust 100-GE transmission,” IEEE J. Lightwave Technol., vol.26, no.1, pp.64-72, 2008.

[35] H. Haunstein, M. Mayrock, "OFDM spectral efficiency limits from fiber and system non-linearities," in Proc. Optical Fiber Communication Conference OFC, no. OThM7, March 2010.

[36] A. Bononi, et al., "Nonlinear signal-noise interactions in dispersion-managed links with various modulation formats," Optical Fiber Technology, vol.16, pp.73-85, 2010.
[37] D. Marcuse, "Bit-Error Rate of lightwave systems at the zero dispersion wavelength,” IEEE J. Lightwave Technol., vol.LT-9, pp.1330-1334, 1991.

[38] Fujitsu factsheet "56 GS/s 8-Bit ADC", preliminary data, Available: http://chais.info, accessed: 1/10/10

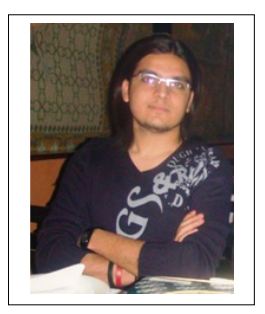

Danish Rafique received the B.Sc. degree from National University of Computer and Emerging Sciences, Pakistan (FASTNUCES) in Electrical Engineering (Telecommunication) (2007), and the M.Sc. degree from The Royal Institute of Technology, Sweden (KTH) in Electrical Engineering (Photonics) (2009). He is currently pursuing his Ph.D. degree with the Photonic Systems Group, Tyndall National Institute and the Department of Electrical and Electronics Engineering, University College Cork, Cork, Ireland. Mr. Rafique is a student member of IEEE and IEICE.

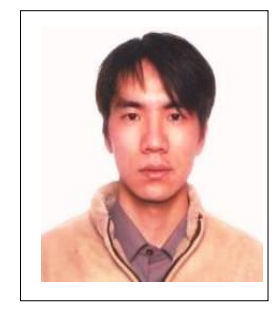

Jian Zhao received the B.Eng. degree from University of Science and Technology of China (USTC) in 2002, and the M.Phil. and Ph.D. degrees from the Chinese University of Hong Kong (CUHK) in 2004 and 2007, respectively. He joined the Photonic Systems Group at the Tyndall National Institute as a Postdoctoral Research Scientist in August 2007. He has published more than 50 technical papers in peer-reviewed international journals and conferences and 2 patents. Dr. Zhao is an Enterprise Ireland principal investigator and acts as a reviewer for IEEE PHOTONICS TECHNOLOGY LETTERS, OSA OPTICS EXPRESS etc. He was the recipient of the First Prize of Outstanding Student Scholarship of USTC.

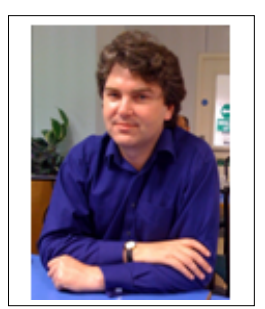

Andrew Ellis received the B.Sc. degree in physics with a minor in mathematics from the University of Sussex, Brighton, U.K., in 1987. He received the Ph.D. degree in electronic and electrical engineering from The University of Aston in Birmingham, Birmingham, U.K., in 1997 for his study on all optical networking beyond $10 \mathrm{Gbit} / \mathrm{s}$. Currently, he heads the Transmission and Sensors Group at the Tyndall National Institute in Cork, Ireland, where he is also a member of the Department of Physics, University College Cork. He has published over 100 journal papers and over 20 patents in the field of photonics. Dr. Ellis is a member of the Institute of Physics and the Institute of Engineering Technology, and is a Chartered Physicist. He acts as a reviewer for IEEE JOURNAL OF LIGHTWAVE TECHNOLOGY and IEEE PHOTONICS TECHNOLOGY LETTERS and as an associate editor for OPTICS EXPRESS. 